\title{
Inhibition and Axial Deviation of Limb Regeneration in the Newt by Means of a Digit Implanted into the Amputated Limb 1
}

\author{
BRUCE M. CARLSON \\ Department of Anatomy, University of Michigan, Ann Arbor, Michigan 48109
}

\begin{abstract}
This research was designed to follow up the observation of Thornton and Kraemer ('51) that regressed, denervated limbs of Ambystoma larvae will not regenerate upon reinnervation if all digits on the limbs were not completely resorbed. The object of this experiment was to determine whether the presence of an apical structure, protruding past the amputation surface, would affect the regenerative process. Both forearms of adult newts were amputated midway between the elbow and the wrist. One limb served as a normal regeneration control, and in the other limb the third digit from the removed hand was implanted in place of the removed radius, so that the three distal phalangeal seg. ments protruded past the plane of amputation. Blastema formation in the experimental limbs was delayed by several weeks as compared with control limbs. Approximately one third of the experimental limbs did not regenerate. The regenerates that did form were strongly deviated $\left(45-90^{\circ}\right)$ radially from the longitudinal axis of the limb. Experimental analysis showed that the delay in regeneration is due largely to the projecting part of the digit. The radial deviation of the regenerates is not due to the digital implant, but rather to the removal of the radius. Trauma alone does not account for this phenomenon.
\end{abstract}

In 1951 Thornton and Kraemer denervated the forelimbs of Ambystoma larvae and demonstrated regression of the entire limbs back to shoulder level if the denervated limbs were slightly damaged in the hand region. When nerves were allowed to grow back into the regressing limbs, regeneration blastemas formed and new limbs developed. These authors also observed that if one or more digits failed to become completely resorbed, despite regression of the zeugopodial and autopodial segments of the forelimb, regeneration did not follow reinnervation of the regressing limb. They were unable to explain the phenomenon, but they did note that the limb remnants appeared unable to organize a regeneration blastema.

This report describes a series of experiments designed to determine the effect of a digit, implanted into a more proximal level of an amputated limb, upon limb regeneration in the newt. The object of these experiments was to ascertain whether or not Thornton and Kraemer's observation on the inhibition of limb regeneration by an unresorbed digit brought to light an example of a general phenomenon that might lead to a deeper understanding of control mechanisms that operate in the regenerating limb.

\section{MATERIALS AND METHODS}

These experiments were conducted upon 560 adult newts Notophthalmus (Triturus) viridescens, collected around Petersham, Massachusetts. The animals were kept in glass bowls at $21^{\circ} \mathrm{C}$ and fed beef liver three times per week.

The basic digital implantation operation will be outlined here. Variations upon this technique will be described for individual experiments along with the results. All animals were anesthetized in $1: 1,000$ MS 222. The forearm was amputated midway between the elbow and wrist, and the radius was then removed. Extirpation of the radius was cleanly accomplished by grasping its distal end and

I Supported by grants from the Damon Runyan Foundation and the Muscular Dystrophy Association. 


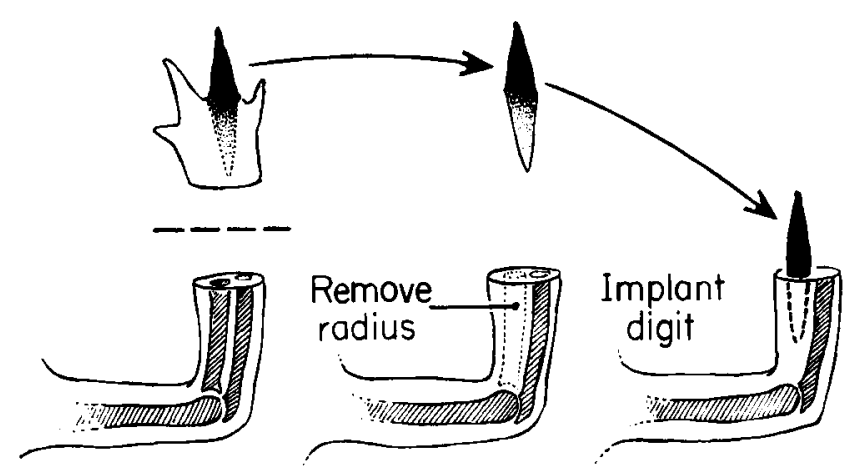

Fig. 1 Diagram illustrating the digital implantation operation.

jerking it sharply. The third digit (including the metacarpal) was removed from the amputated hand and was implanted (with normal dorsoventral and anteroposterior orientation) into the site formerly occupied by the radius (fig. 1). No sutures were required to hold the implanted digits in place. After the operation the animals were placed in cold water $\left(4-6^{\circ} \mathrm{C}\right)$ for three days, after which time they were brought back to room temperature. Animals kept in the cold for longer periods of time did not always regenerate according to a normal timetable, and several experimental groups had to be repeated because of this.

For radioautography, donor animals were given IP injections $(2.0 \mu \mathrm{Ci} / \mathrm{gm})$ of thymidine-methyl- $\mathrm{H}^{3}$ (New England Nuclear). Digits were removed for transplantation four hours later. Five-micron tissue sections were dipped in Kodak NTB-3 emulsion, stored in light-tight boxes for two weeks and developed with Kodak D-19.

In all experiments involving $x$-irradiation, the irradiation was delivered by a Westinghouse Coronado machine at $150 \mathrm{kv} ; 15 \mathrm{ma}$; FSD-28 cm; filter $-0.5 \mathrm{~mm} \mathrm{Cu}+1.0 \mathrm{~mm} \mathrm{Al}$; rate $-435 \mathrm{r} / \mathrm{min}$.

Many regenerates were fixed in Bouin's fluid, serially sectioned and stained with hematoxylin and eosin, Heidenhain's aniline blue stain for connective tissue or Hsu's ('71) modification of the Palmgren silver stain for nerves.

Other regenerates were prepared as whole mounts for skeletal staining, following a modification of the toluidine blue-alizarin red technique outlined by Burdi ('65). In newt limbs cartilage stained best in $0.12 \%$ toluidine blue in $70 \%$ alcohol and bone in 16 drops of $0.1 \%$ alizarin red $\mathrm{S} / 100 \mathrm{ml}$ of $5 \% \mathrm{KOH}$.

\section{RESULTS}

\section{The inhibition of regeneration by an implanted digit}

\section{Series 1. Simple digital implantation}

In this series, comprising over 150 animals, one arm of each animal was amputated midway between the wrist and elbow as a normal regeneration control. The other arm was similarly amputated, but the radius was removed and a digit was implanted in its place as described in MATERIALS AND METHODS.

\section{A. Gross observations}

During the first couple of days postoperatively, epithelial migration covered the amputation surface, and epidermal continuity was established between the skin of the stump and that covering the digital implant. The digital implant remained disconnected from the circulating blood for about five or six days, and during that time healthy implants were noticeably less translucent than normal dig. its. Eighty-five to ninety percent of the digital implantations were successful. Within a few days after the return of circulating blood, the appearance of most implants resembled that of normal digits.

During the early weeks after digital implantation, an average of $50 \%$ of the cross section of the amputated limb surface was covered by a typical early wound epidermis. Constriction of the epithelialized wound surface by old skin did not occur. Typically, the wound epidermis of experimental limbs became thicker and less transparent than nor. mal, and for 50 days a smooth pattern of wound healing occurred.

Beyond 50 days, one of two courses followed. A substantial percentage of the limbs bearing 
implanted digits never regenerated. In an early series of 26 limbs, $11(42.3 \%)$ failed to regenerate (fig. 3 ). In these cases the wound epithelium developed into full thickness skin. Six long term non-regenerating limbs were reamputated through the ulnar side, leaving the digital implant intact. Regeneration did not occur after reamputation.

In the remaining limbs, regeneration occurred from the ulnar side. Full dedifferentiation began late, often 40 to 50 days after amputation, and blastema formation quickly ensued. The blastemas were rarely typical in form, but rather began as an indistinct swelling around the ulnar sides of the base of the implanted digit. Some limbs that appeared to be undergoing dedifferentiation stabilized and underwent no further regenerative changes. Others that had begun to form blastema-like structures suddenly stopped progressing, and the outgrowth either disappeared or became smaller and remained as a small projection. The remaining limbs formed hypomorphic regenerates with from one to three digits. Four-digit regenerates did not form in limbs bearing intact digital implants. Two-thirds of the regenerates were single digit spikes.

A consistent and unusual feature of the regenerates was their orientation. The regenerates in these experiments were sharply deviated toward the radial side of the limb. The angles of deviation commonly ranged from $45^{\circ} \cdot 90^{\circ}$.

\section{B. Histological observations}

During the early phases of wound healing and demolition, there was virtually no difference between control limbs and limbs bearing digital implants (figs. 8, 9). The digital implants healed in place with remarkably little cellular reaction from the surrounding stump tissues, and normally there was no ingrowth of wound epidermis alongside the implant into the deeper tissues of the stump (fig. 9).

By the period of dedifferentiation the digital implants were stabilized, and essentially no scarring or other tissue reactions surrounded the deep part of the implants. Although osteoclastic erosion of the metacarpal element of the implanted digit occurred, a typical dedifferentiative response was not noted in the digit itself. Throughout the dedifferentiative phase a typical wound epidermis, not underlain by dermis or histologically visible membranes, was prominent over the ulnar surface of the limb. During this period the wound epidermis of normally regenerating limbs consistently undergoes a thickening. The wound epidermis of the experimental limbs was also typically thickened in areas, but the degree and extent of thickening was more variable than that seen in control limbs.

During the early phase of the dedifferentiative reaction, the histological picture of both the bone and the soft tissues of the limb stumps was similar in both normal and experimental limbs. By the end of the period of dedifferentiation (early in the third week in these experiments), a divergent pattern of development between normal and experimental limbs was emerging. Whereas in control limbs the distal soft tissues were in the peak of the dedifferentiative reaction and early condensations of blastemal cells were accumulating beneath the wound epidermis, limbs bearing digital implants showed signs that the early regenerative process was beginning to slow down. Although the soft tissues still presented the histological appearance of dedifferentiation, blastemal cells did not accumulate beneath the wound epidermis to any significant degree. Instead, early traces of tissue healing responses began to appear. Equivalent amounts of osteoclastic activity were present in control and experimental limbs.

By the middle of the fourth week the control limbs possessed well-developed, but undifferentiated blastemas (fig. 10). The experimental limbs still possessed a prominent area of wound epidermis, which was commonly thickened. Dedifferentiation still persisted in the deeper tissues of the stump, but in most limbs there was still no distal accumulation of blastemal cells (fig. 11). The digital implants were morphologically stable and were well integrated with the tissues of the stump. Later in the fourth week and early in the fifth some experimental limbs contained small distal accumulations of blastemal cells whereas in others the scarring and tissue healing reaction was more prominent.

The difference between normal and experimental limbs was accentuated during the period of blastemal differentiation (during the fifth and sixth weeks after amputation). In contrast to the control regenerates (figs. 12, 14 , 16), which demonstrated increasingly more complex levels of morphogenesis, the contralateral limbs bearing digital implants presented a picture of little or reduced epimorphic regenerative activity. The more ac- 
tive limbs (fig. 15) had a thickened wound epidermis on the ulnar side of the implanted digit. Beneath the epidermis was a small to moderate accumulation of typical blastemal cells, and deep in the stump tissue dedifferentiation was still in evidence. In a larger proportion of cases, the limb stump was characterized by the differentiation of a terminal cap of cartilage around the cut end of the ulna (fig. 13). Between the cartilagenous cap and the epidermis was a layer of dense collagenous connective tissue in addition to the newly differentiating dermal elements of the skin. The digits in all long term limbs were well integrated with the limb stump (figs. 13, 17).

Long term experimental limbs could again be subdivided into two categories - those that exhibited evidence of epimorphic regeneration (fig. 15) and those that did not (figs. 13, 17, 18). Nevertheless, all limbs in these two categories were organized in roughly the same manner. The implanted digits remained in good shape and were morphologically stable, without evidence of ongoing regenerative activity. Likewise, the ulnar borders of virtually all limbs presented the appearance of a completed tissue regeneration process, with a prominent fibrocellular mat under what was originally wound epidermis (fig. 18). The differentiation of new tissue took place principally in the region between the ulna and the digital implant. When epimorphic regeneration occurred, one or two shortened digits could be seen. Small rods of unjointed cartilage were buried within the soft tissues of the stump in other limbs, and occasionally only small, irregularly shaped masses of cartilage capped the ulna. The association between the tissue healing response over the lateral side of the ulna and the radial direction of regenerative growth was striking in the histological preparations (figs. 13, 18), but at this point it is not possible to say if the ulnar scar is a cause or an effect of the radial direction of regenerative growth.

\section{Autoradiographic observations}

An important piece of information needed to narrow down possible mechanisms responsible for the inhibition of regeneration in limb stumps bearing digital implants is the origin of the wound epidermis that covers the amputation surface. In order to determine whether the wound epidermis arises from that of the stump or the implanted digit, a reciprocal transplantation experiment of isotopically labelled digits to non-labelled limbs and vice versa was performed.

Four donor newts were labelled with tritiated thymidine as described in MATERIALS AND METHODS. Four hours after injection of the isotope digits from the labelled donors were implanted into 16 freshly amputated recipient forelimbs as in typical digital implantation experiments. Then both forearms of each donor newt were amputated, and unlabelled digits were implanted into each stump. In the series with labelled digits in unlabelled arms, two limbs were fixed for autoradiographic analysis each day for eight days. Two labelled limbs bearing normal digital implants were fixed at $2,3,5$ and 7 days after implantation. In the labelled limbs with unlabelled implants, there were labelled cells throughout the wound epidermis whereas the wound epidermis in the normal limbs bearing labelled implants was unlabelled. It is not possible to rule out a contribution to the wound epidermis of occasional unlabelled cells from the implanted digits, but the results of both series of experiments demonstrate that at least the majority, if not all, cells of the wound epidermis arose from the epidermis of the stump and not from the implanted digit.

\section{Experimental analysis of inhibition of regeneration by an implanted digit}

In order to understand the nature of the inhibition or retardation of limb regeneration by an implanted digit, a series of experiments was designed to dissociate non-specific traumatic effects from a non-traumatic biological phenomenon. Right and left forelimbs were treated differently and the difference in days of the appearance of a fixed stage in regeneration (2-digital primordia) was noted.

In the first group (fig. 2, upper), a digit was implanted into the left forearm in each of 16 animals, and the right arms were amputated at the same level as the left. No regeneration occurred in five $(31 \%)$ of the experimental limbs. In the remaining animals, the formation of digits in the left limb was delayed by a mean of 21.1 days over that in the right. Such inhibition and delay was representative of the reactions of the other animals (total of 96) in which this design was followed. The remainder of the animals, however, were not examined at daily intervals and the mean delay could not be accurately recorded. 


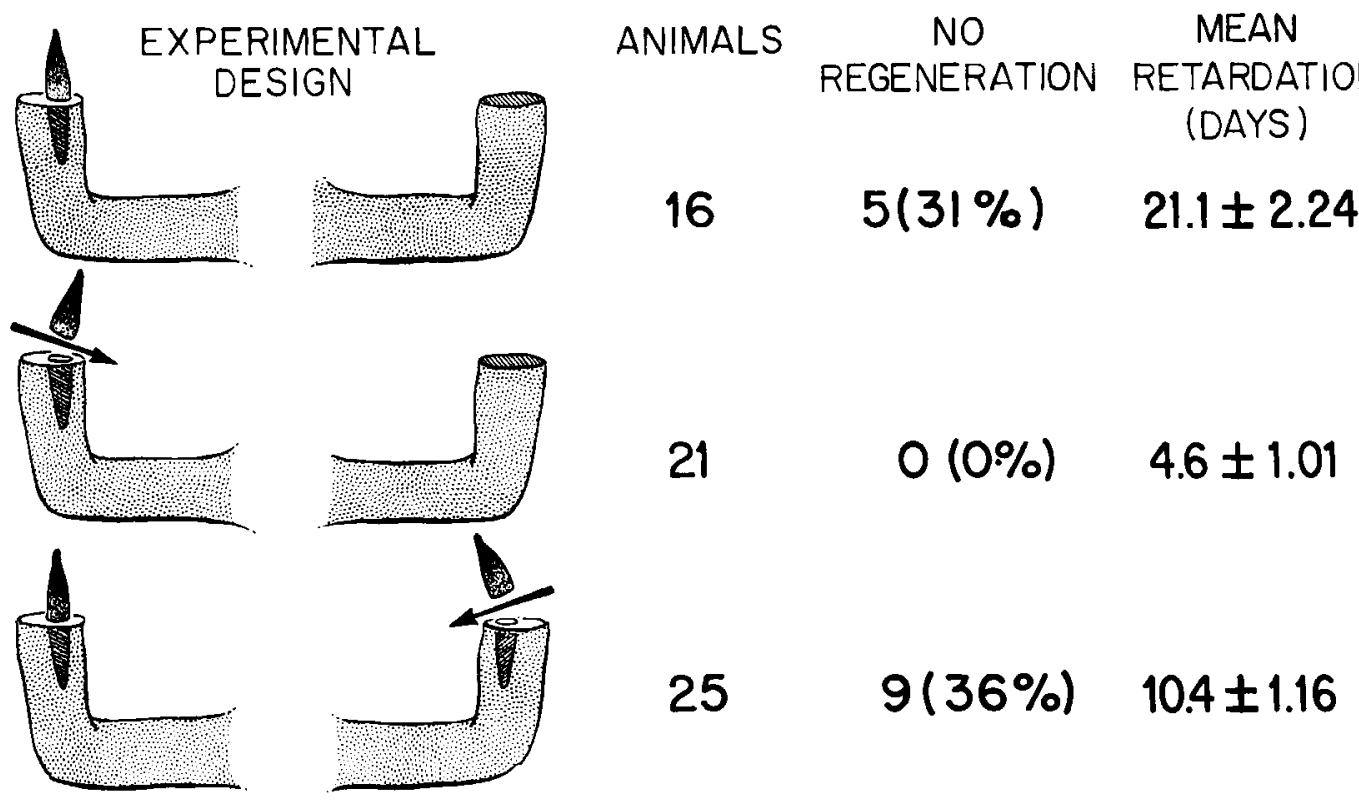

Fig. 2 Experimental design and results of experiments demonstrating the inhibitory effects on regeneration of digital implants. The "No Regeneration" column refers to the left limbs in each series. "Mean Retardation" refers to retardation of regeneration of left limbs as opposed to the right limbs in each series.

The next experiment (fig. 2, middle), was designed to determine the degree of delay in regeneration that could be attributed to the deep, implanted part of the digit. In 21 animals, one forearm was amputated midway between elbow and wrist as a normal regeneration control. The contralateral arms were amputated and digits were implanted in place of the radii as usual. Immediately after implantation, the digits themselves were amputated at the level of the amputation surface of the forearm. In this experiment any delay in regeneration over that in the control limbs can be attributed to the trauma of implantation or to some intrinsic property of the basal part of the digit.

Histologically, the early stages of regeneration did not differ significantly from those seen after the implantation of entire digits except that the entire limb surface was covered with a wound epidermis. In contrast to limbs bearing implants of entire digits, regeneration occurred in all experimental limbs, with a mean delay of 4.5 days between the formation of digital primordia in the experimental limbs over that in the control. Despite the lack of a projecting portion of the distal implant, regenerates on the experimental limbs were sharply deviated from $45-90^{\circ}$ radially from the longitudinal axis of the limb.

This experiment shows that the presence of the basal part of the implanted digit accounts for less than a week's delay in regeneration. In addition to the fairly consistent absence of regeneration in about one-third of the limbs with entire digital implants, a delay in regeneration of about two weeks appeared to be related to the projecting part of the digital implant. This was tested directly by the next experiment.

In 25 newts, both forearms were amputated midway between the elbow and wrist, and in each arm digits were implanted in place of the radius in the usual fashion. On one limb the digit was left intact and on the other limb the digit was cut off level to the amputation surface (fig. 2, bottom). Thus the only difference between the two limbs was the presence of the projecting part of the digit on one side.

Nine $(36 \%)$ of the limbs with entire digital implants failed to regenerate whereas only one limb (4\%) bearing an amputated digit did not regenerate. Among the 15 animals in which both limbs regenerated, a mean delay of 10.4 days was associated with the presence of an entire digital implant as opposed to a 


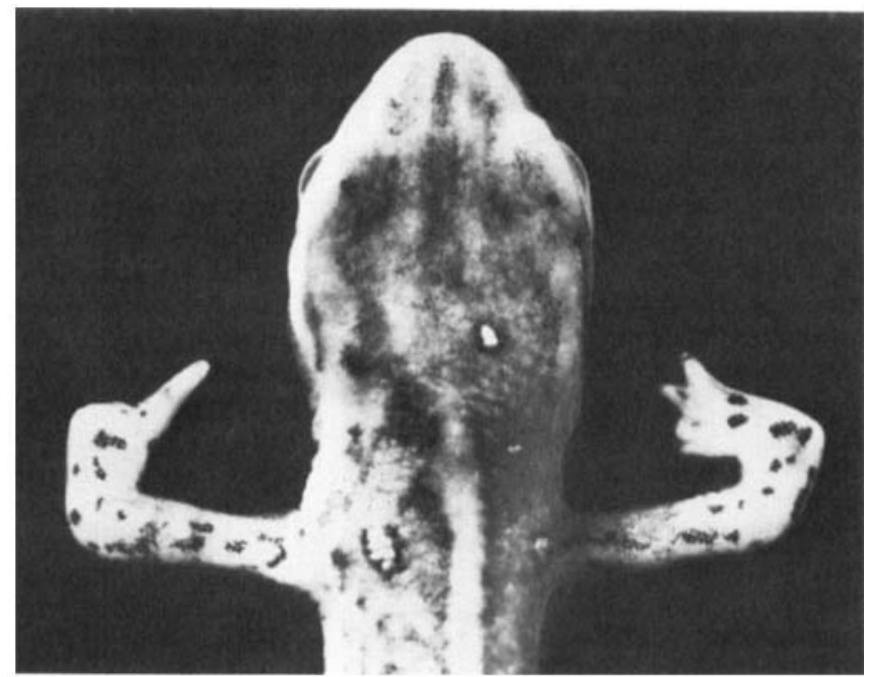

Fig. 3 Newt nine months after bilateral forearm amputation. Left arm, the radius was removed immediately after amputation and the left third digit was implanted into its place. The digital implant remained intact and projects prominently from the amputation surface of the forearm. No regeneration of the arm occurred. Right arm, the radius was removed immediately after amputation. The right third digit was implanted in its place and immediately amputated at the level of the limb amputation surface. A somewhat abnormal 4-digit regenerate has grown out at almost a right angle radial to the normal longitudinal axis of the limb.

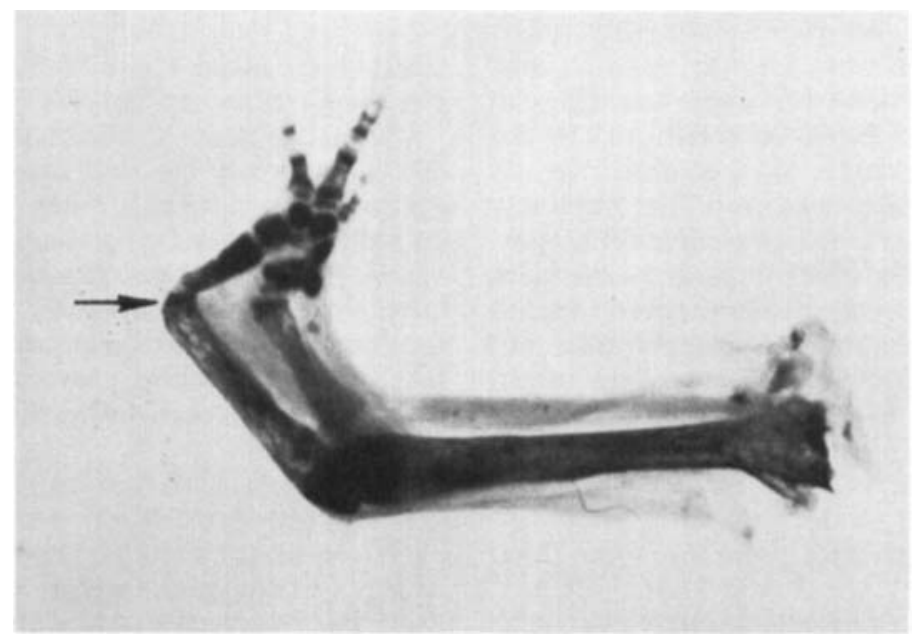

Fig. 4 Ventral view of a skeletal preparation of an 11-month regenerate arising from a limb bearing an amputated digital implant. The regenerate grows out from the limb stump at a very sharp angle (arrow). This limb is equivalent to the right limb of figure 3.

digit amputated level with the wound surface of the limb (fig. 2, bottom). The regenerated limbs were typical of what was seen in the previous experiments (figs. 3,4). This experiment demonstrated that the delay in regeneration associated with the projecting portion of the digital implant was somewhat less than two weeks. The results of this experiment, along with the previous two, suggests that the mechanism of inhibition of regeneration is associated with some way in which the projecting digit interferes with the wound epidermis or with events occurring at the wound surface.

During the course of the preceding experiments, it was noticed that when the im- 


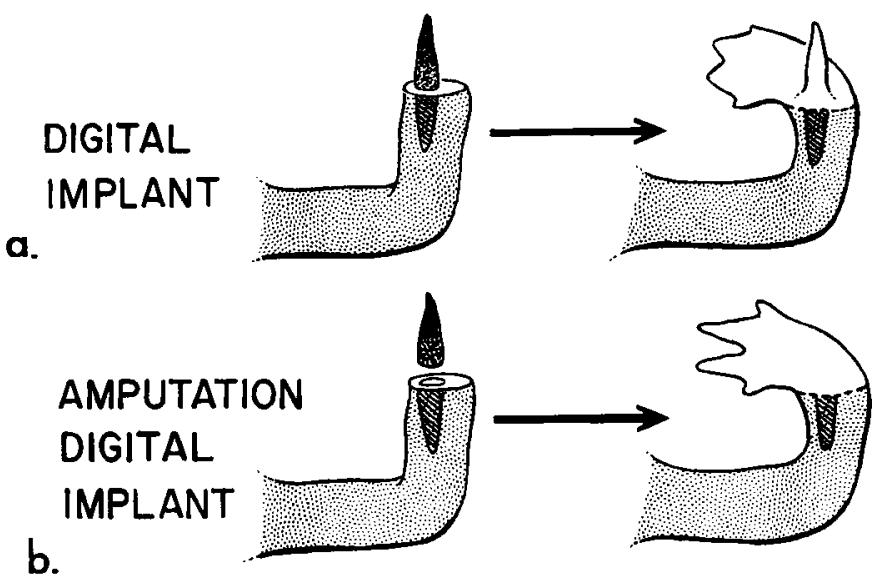

Fig. 5 Two experiments demonstrating (a) the radial deviation of regenerates on limb bearing digital implants and (b) that the radial deviation is not related to the projecting part of the digit.

planted digits occasionally became necrotic and distal segments fell off, the remainder of the digital stump appeared to be covered over by a clear wound epidermis. Limb regeneration, little delayed over normal, soon followed. An experiment was designed to test whether or not the inhibitory effect of the implanted digit required the presence of the terminal phalangeal segment.

In ten animals both forearms were amputated, and digits were implanted in the usual manner. The terminal phalangeal segment was removed from the right digital implants. Both implants in eight animals remained healthy enough on valid comparison. In two animals regeneration failed to occur on one limb (one right and one left). The remaining six animals showed no significant difference in the rate of regeneration between the limbs with intact or with terminally amputated digits. It was concluded that as long as the base of the protruding digit remains healthy and projects noticeably from the amputation surface, the presence of the terminal segment of the digit is not required to produce the inhibitory effect on regeneration.

\section{Radial deviation in regenerates}

After the initial observation of radially deviated regenerates in limbs with digital implants (fig. 5a), radially deviated regenerates were consistently seen to arise from limbs with digital implants cut off at the level of the limb amputation surface. This demonstrated that the deviation phenomenon, in contrast to the inhibition of regeneration, was not related to or dependent upon the projecting portion of the implanted digit. Thus it appeared that two fundamentally different processes were combining forces to produce the retarded, radially deviated regenerates in the original digital implant experiment. Several groups of experiments were performed to ascertain some of the factors that play a role in producing the deviated regenerates.

The first experiment was designed to determine whether or not the implanted digit played any role whatsoever in the formation of radially deviated regenerates. Twenty limbs were amputated at mid-forearm level and the radius was removed in the usual fashion. No digital implant was placed into the limb stump (fig. 6a). With a couple of exceptions, the regenerates were deviated 45 . $90^{\circ}$ radially.

The role of trauma itself was investigated in a series of $\mathbf{5 2}$ amputated forearms in which the radius was removed in the usual fashion, but then replaced (fig. $6 \mathrm{~b}$ ). The orientation of the majority of regenerates was completely normal, but in five cases radial deviations of $5-15^{\circ}$ were present.

In 20 newts, both arms were amputated at mid-forearm level. As a control, the radius of one limb was removed and replaced as previously described (fig. 6b). The results of that experiment were duplicated in the controls, and the regenerates grew out normally or were radially deviated less than $15^{\circ}$. On the contralateral limbs, the radii were removed, placed in saline, $\mathrm{x}$-radiated with $2,000 \mathrm{r}$ and orthotopically replaced within one and one- 


\section{REMOVE RADIUS}

a.

\section{REMOVE 8 REPLACE RADIUS} b.

\section{X-RAY RADIUS \& REPLACE}

c.

\section{BOIL RADIUS \& REPLACE d.}

Fig. 6 A series of experiments demonstrating the relationship between removal of the radius and other manipulations on the radius and the orientation of the longitudinal axis of the regenerate with respect to that of the stump.

half hours of removal (fig. 6c). Healing was uneventful and the regenerates from limbs bearing irradiated radii were virtually identical with those arising from the contralateral control limbs.

In another experiment, involving 83 newts (fig. 6d), both limbs were amputated through the mid-forearm. On one side the radius was removed and immediately replaced as the standard damage control. Normally oriented regenerates appeared on the control limbs. The radii were also removed from the contralateral limbs, but were placed in boiling saline for five minutes before being reimplanted. The boiled bones appeared to be treated like foreign bodies by the limb stump, and many of them were extruded over a period of several weeks. Thirty-nine limbs were suitable for analysis, and of these $85 \%$ produced regenerates that were radially deviated by more than $45^{\circ}$. Even in these cases, the bone was almost always ultimately extruded during the course of regeneration although often not until very late in the regenerative process, when the regenerate was already strongly deviated.

In some of the previous experiments, the rapid tissue healing reactions of the soft tissues and skin on the lateral position of the tip of the ulna necessitated a separate control ex. periment in order to ensure that the radial deviation of regenerates was not due to a pinching off of the ulnar wound surface by old stump skin. This would have the effect of forc. ing the blastema to grow out at an angle to its normal axis of growth.

In 12 newts both arms were amputated slightly proximal to the wrist (fig. 7). The radii were removed from the right arms in the usual fashion. Then a $1.5-\mathrm{mm}$ cuff of skin was 


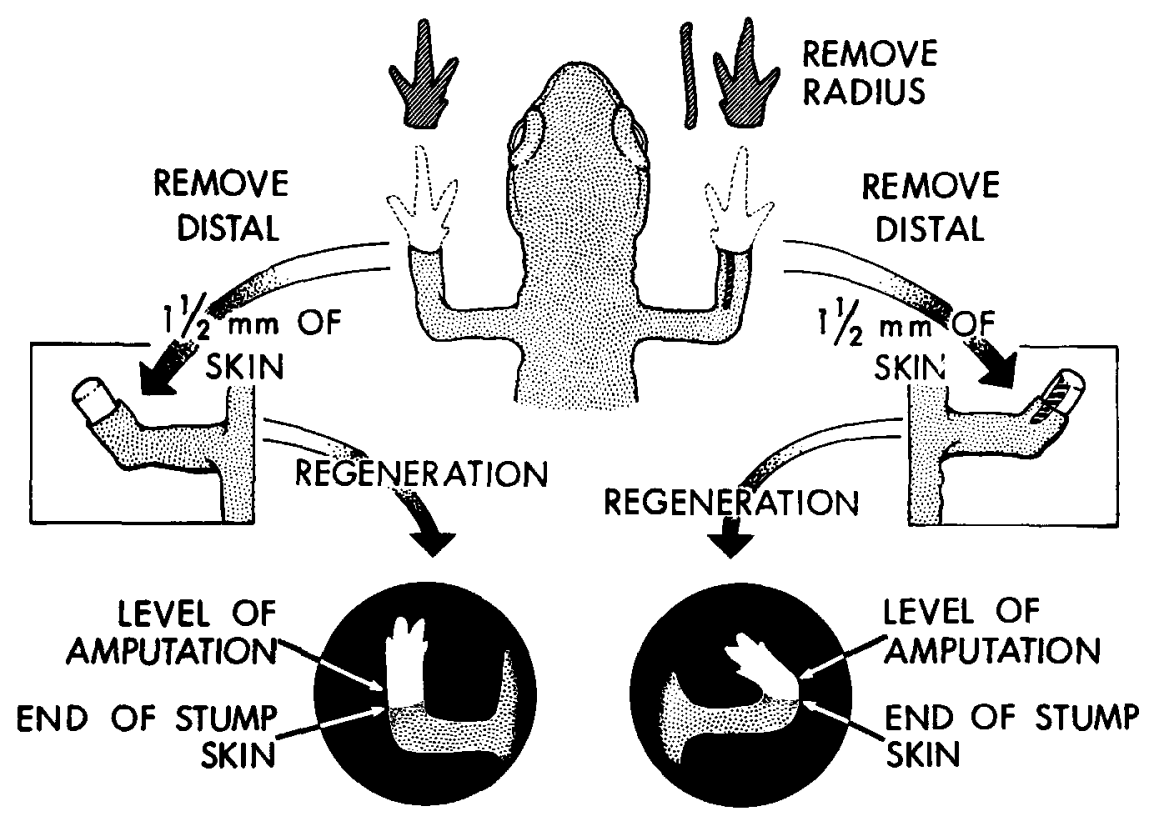

Fig. 7 Design and summary of an experiment demonstrating (right arm) that the radial deviation of regenerates arising from limbs lacking the radius is not due to encroachment of stump skin upon the ulnar side of the amputation surface.

removed from the end of both right and left limb stumps, and the animals were placed in a cold room for a few days before being returned to normal room temperatures. It was not possible for stump skin to be pulled over the amputation surface. The denuded tips of the limb stumps became epithelialized and regeneration ensued.

The left control limbs produced normal regenerates with no or occasionally $5-10 \%$ radial deviation. Two of the experimental limbs exhibited little axial deviation but the remainder were radially deviated from $40-85^{\circ}$, demonstrating that the phenomenon of radial deviation is not an artifact caused by selective covering of part of the amputation surface by stump skin.

Another potential artifactual cause of the radial deviation in these experiments is the possibility that removal of the radius removes a mechanical support for the nerve which might itself become strongly deviated in a radial direction. Deviation of the nerve could potentially result in the formation of an eccentric apical epidermal cap and then a deviated blastema. To test this possibility, both limbs of 39 animals were amputated through the mid forearm and the radii of the right arms were removed. At intervals from 1 to 21 days after amputation both control and experimental limbs were removed, fixed in Bouin's and stained with Palmgren's silver stain to chart the pathway of the distal nerve stumps and fibers.

No consistent or substantial radial deviation of nerves was observed in the limbs from which the radius was removed. Figure 24 shows a typical example of an experimental limb, possessing both a straight nerve trunk and a non-deviated apical epidermal cap. Thus there is no apparent causative relationship between radially deviated regenerates and the orientation of the peripheral nerve stumps.

\section{Removal of other stump bones}

The pronounced radial deviation of regenerates toward the side of the limb from which the radius was removed prompted some experiments to see if a similar deviation of regeneration in the direction of removal of other bones would occur.

Twenty forelimbs were amputated and the ulna was removed instead of the radius. Only one regenerate was significantly deviated ulnarly $\left(60^{\circ}\right)$. The remainder of the regenerates grew out straight or with a $10-20^{\circ}$ ulnar deviation. 
In 32 animals each hindlimb was amputated midway through the zeugopodial segment. The tibia was removed on one side and the fibula from the other. A pronounced tibial deviation $\left(45-90^{\circ}\right)$ occurred in regenerates arising from the stumps without the tibia. Deviations of similar magnitude, but in a fibular direction were seen in all regenerates except one ( $15^{\circ}$ deviation) from stumps lack. ing a fibula.

\section{Retardation of rate of growth in radially deviated regenerates}

A potential cause of or contributing factor to the retardation of regeneration from limb stumps bearing digital implants could be some retardation associated with the great radial deviation of the regenerates that did form. In order to control for this, both forelimbs on 20 newts were amputated midway between the elbow and wrist. One limb served as a normal regeneration control whereas the radius was removed from the other. Regenerates from the control limbs formed digital primordia approximately three days earlier than the strongly deviated regenerates arising from limb stumps without the radius.

As a control for effects of trauma, both forearms of 20 newts were amputated midway between the elbow and wrist. The radius was removed from the experimental limbs as usual, and on the control side the radius was removed, but then immediately replaced. In this experiment both experimental and trauma control limbs formed digits at about the same time despite the fact that the regenerates from the experimental limbs were deviated and those of the trauma controls were not. These experiments demonstrate that only a slight delay, at most, in the rate of regeneration could be attributed to the axially deviated growth pattern of the regenerates.

\section{DISCUSSION}

The experiments described above have brought to light two means by which profound alterations in the limb regenerative process can be produced. One is the inhibition or retardation of regeneration from the midforearm level by means of a digit implanted into the limb stump. The other is the production of axially deviated regenerates by removal of the radius in an otherwise normal forearm stump. The mechanism accounting for neither of these disturbances in regeneration is understood. Both are highly repeatable phenomena, and control experiments have shown that these disturbances in regeneration cannot be ascribed to inconsistencies or unexplained vagaries in technique. It is likely that these phenomena are independent, because experimental analysis showed that the inhibition of regeneration could be attributed to the presence of projecting portions of the digital implant whereas axial deviations of regenerates don't require the digit at all, but can be obtained by removing the radius from the limb stump.

\section{Retardation of regeneration by digital implant}

In their experiments on regression of injured denervated limbs and subsequent regeneration following re-innervation, Thornton and Kraemer ('51) concluded that when differentiated distal structures (i.e., digits) are present in a regressed limb, blastema formation and subsequent regeneration does not occur.

During the next decade, Rose ('57, '62), largely on the basis of experiments conducted on Tubularia, formulated the concept of distal control, according to which the most distal structure in a regenerating system permits the differentiation only of structures more proximal to them. Indirect evidence in favor of this system of control in the regenerating amphibian limb was presented in a review (Rose, '62).

In 1967, Shuraleff and Thornton reported experiments designed to test Rose's concept of distal dominance in the regenerating hindlimbs of the axolotl. They amputated legs through the mid-femoral region and transplanted a foot over part of the amputation surface. In their experiments inhibition of regeneration did not occur. They were carried out along the regenerating limb until they reached a level homologous with the most proximal level of the foot graft. Although the authors did not discuss their findings, the results of their experiments do not necessarily constitute a refutation of Rose's concepts because a large number of variables remained untested.

The recent demonstrations of intercalary regeneration in urodeles (Iten and Bryant, '75; Stocum, '75) have established that regeneration can occur between a proximal amputation surface and a regeneration blastema taken from a more distal level if the transplanted blastemas are not too highly developed. In Ambystoma larvae, Stocum ('75) 
showed that intercalary regeneration did not occur when advanced 4-digit regenerates were transplanted, whereas intercalary regulation did occur proximal to notch stage blastemal grafts. On the other hand, Iten and Bryant ('75), working with adult newts, obtained intercalary regulation between proximal limb stumps and distal blastemas in the early digits stage.

Why a differentiated digit, implanted into a fresh amputation surface, significantly retards or inhibits limb regeneration is not known although there are several possible directions in which explantations can be sought. On the basis of the control experiments, there is little reason at this point for considering surgical trauma or a disruptive influence of the deep part of the implanted digit as a major factor. Instead, one must look to the projecting part of the digital implant for the answer.

According to one option, the implanted digit could act as an apical inhibitor as postulated by Rose ('62). A problem with this interpretation is that in two-thirds of the cases the regeneration of hands and digits, albeit abortive, ultimately does occur. Similarly, regen. eration regularly occurs when part of a hand or foot is amputated, leaving the remainder of the digits intact (Tornier, 1896). One could postulate that in the distal part of the limb these are multiple centers of apical inhibition corresponding to the digital rays and that complete inhibition does not occur if the wound surface and the remaining $\operatorname{digit}(s)$ is out of line. The phenomenon of intercalary regeneration, however, weakens the argument for apical inhibition as a control mechanism in amphibian limb regeneration. These digital implantation experiments provide no evidence for or against an hypothesis of chemical inhibition of regeneration by an apical structure.

Because both the species and the experimental systems were different, it is hard to compare the lack of effect upon regeneration of the foot graft in the experiments of Shuraleff and Thornton ('67) and the retarding of inhibiting effect exerted by digital implants in the work described here. One significant variable is the mode of attachment of the graft to the amputation surface. Shuraleff and Thornton ('67) apparently placed their foot grafts on the amputation surface and allowed them to heal in place without sutures (Connelly, personal communication). In con- trast, the digital implants in the newt were tightly fixed to the limb stump by the deeply implanted metacarpal element. Firm fixation of the digital implant to the stump might conceivably interfere with intercalary regeneration, but it would be less likely to affect the regenerative process that of ten ultimately takes place on the ulnar half of limbs bearing digital implants.

A possibility worthy of close examination is that the projecting part of the digital implant interferes with regeneration not by anything intrinsic to the digit itself, but by some interference with the normal spatial pattern of epithelial healing of the amputation surface. It is well known that in normal regeneration the presence of a wound epidermis is a necessary condition for blastema formation (Mescher, '76; Stocum, '75; Thornton, '68). In the past, most workers in regeneration have considered the wound epithelium to be a morphogenetically neutral structure. More recently, however, it has been recognized that the pattern of epithelial migration, particularly the point at which migrating epithelial sheets from opposite sides of the limb meet, may be of critical importance with respect to fulfilling necessary morphogenetic boundary conditions. Lheureux ('75) in urodeles and Chandebois ('76) in planaria have attributed great significance to the meeting of tissues from opposite sides of the amputation surface with respect to the initiation of regeneration. If a similar mechanism can be confirmed in the newt, the digital implant could be looked upon as a means of preventing or retarding such a meeting. According to a similar line of reasoning (Bryant and Iten, '76), the digital implant may prevent the immediate filling in of the "full circle" of positional values that is postulated to be a necessary pre-condition for outgrowth. It is possible that the delay in regeneration of limb stumps bearing implanted digits represents the time required to reorganize the morphogenetic relationships required for outgrowth to occur.

\section{The formation of axially deviated regenerates}

The radial deviation of regenerates was due to removal of the radius in the limb stump rather than the presence of a digital implant. Operative trauma to the radius or surrounding tissues is a most unlikely cause of radial deviation in view of the experiments reported here.

Another possibility is that the amputation 
surface in a forelimb stump lacking a radius is no longer perpendicular to the long axis of the limb. According to "Barfurth's rule" (Morgan, '01: pp. 44-52), a regenerate grows out perpendicularly to the wound surface. Thus, a regenerate growing out from an oblique amputation surface would be deviated away from the long axis of the stump. A limb stump lacking one of the zeugopodial elements does have a degree of obliquity to its amputation surface (up to $10-20^{\circ}$, but Barfurth's rule cannot account for the frequent regenerates that grow out at right angles to the long axis of the limb stump or are even directed slightly toward the base of the limb $\left(100^{\circ}\right.$ or more radial deviation). In addition, axially deviated regenerates that grow out from oblique amputation surfaces commonly straighten out nearly completely by the end of their growth period.

A similar possible mechanism is the deviation of the cut end of nerves in a radial direction, thus causing an eccentric blastema to form. Histological examination of many limbs did not reveal significant deviations of the cut ends of nerves.

The experiment on reimplantation of boiled radii was difficult to interpret. The regenerates were typically radially deviated, suggesting that boiling destroyed some property of the radius that keeps normal regenerates growing out in line with the long axis of the limb. Two main interpretations of this experiment are possible. One is that boiling destroyed some intrinsic property of the radius and that the physical presence of the boiled radius was insufficient to prevent radial deviation. The other is that from a physical standpoint the boiled radius is not the equivalent of a viable bone. In virtually all cases, the boiled bone was apparently considered to be a foreign body by the limb stump and was eventually extruded from the limb. Often, how. ever, the regeneration blastema was strongly deviated before extrusion of the radius occurred. Because of the unanswered questions concerning the degree and kind of integration of the boiled bone with the surrounding stump tissues, critical inferences should not be made from this experiment.

From morphological observations alone, one might be tempted to attribute the radial deviation of regenerates to the riding up of stump skin over the ulnar half of the limb, thus forcing the regenerate to grow in a radial direction. Tissue healing responses over the ulna are certainly prominent. There are su. perficial similarities between the deviated regenerates reported here and those pictured by Mescher ('76, his fig. 6), who obtained strongly deviated regenerates in newts after sealing the amputation surfaces with partial skin flaps. The regenerates in Mescher's experiments, however, usually showed imperfect morphogenesis whereas the deviated regenerates reported after radial removal were otherwise almost always morphologically normal. This possible explanation was eliminated by the experiment in which the distal skin was stripped from a radius-less stump. The appearance of radially deviated regenerates in this case also leads to the conclusion that the tissue healing reactions over the postaxial side of the ulna are probably symptoms and not causes of the axial deviation.

Axial deviation of regenerates toward the side of the limb from which one of the paired zeugopodial bones was removed is not unique to forelimbs lacking a radius, for similar deviations occurred consistently in legs from which either the tibia or fibula was removed at amputation. Surprisingly, significant ulnar deviation does not occur in regenerates arising from forearm stumps lacking an ulna. No explanation can presently be made for the lack of axial deviation in the latter case. It is noteworthy that Goss ('57, his fig. 4) illustrated a newt forearm regenerate with a pronounced ulnar deviation. In this case, however, the ulnar half of the limb was removed rather than the ulna alone.

Deviation of regenerates from the longitudinal axis is commonly seen in half-limb or limb splitting experiments. Weiss ('26) and Lomovskaya ('48) have each illustrated hand regenerates from the ulnar halves of longitudinally split limbs, and these deviated sharply in a radial direction. In both cases, the regen. erates growing from the radial halves of the limbs were illustrated as simple spikes. In his half limb experiment, conducted on both the arms and legs of newts, Goss ('57) found deviation of the regenerates toward the side of the limb that was removed. During the course of her experiments on the effect of bone removal in the stump on morphogenesis of regenerates, Bischler ('26) found frequent instances of deviation of the entire regenerate toward the ulnar side from which the bone had been removed. The common factor in all of these experiments is that the regenerates grew out toward the side of the limb from which some structure or tissues had been re- 
moved. In all of these defect experiments it is possible that the altered wound surface results in an abnormal pattern of epithelial healing, such as displacement of the site of joining of the advancing edges of the wound epidermis. This might result in the setting up of an eccentric focus of regenerative activity.

Some indication that the fundamental morphogenetic defect demonstrated in these regeneration experiments might be a general one can be gained by observations on congenital limb defects. Commonly, when one of the zeugopodial bones is reduced or missing, the hand or foot is strongly deviated toward the side of the limb with the missing bone. A good example of this is seen in figure 102-14 from Warkany's book ('71), which shows clubfoot associated with tibial shortening in a thalidomide-treated rabbit embryo.

An interesting example of axial deviation during regeneration was recently reported by Globus and Liversage ('75), who implanted dorsal root ganglia into the base of blastemas grown in culture. They noted that "a conspicuously dense population of blastema cells accumulated around the implanted ganglion, and subsequent orientation of pro-cartilage formations favored the eccentrically implanted neurons." Comparison of their figure 14 and figures 13 and 18 of this paper reveals a striking similarity in morphology. Because the main mass of nerves in the forelimb lies between the radius and the ulna, removal of the radius would not bring a major nerve mass any closer to the ulna than before, but the morphological balance of nerves and skeleton would be altered.

At this point it is only possible to say that axial deviations of regenerating or developing limbs toward an area of tissue deficit is a common and perhaps general phenomenon. The next major step in uncovering the underlying mechanism should be to dissociate purely mechanical effects that lead to disturbed morphogenesis because of a change in geometry of the stump from morphogenetic tissue interactions that might be altered by tissue removal or by displacement of other tissues subsequent to tissue removal.

\section{ACKNOWLEDGMENTS}

I wish to thank Mrs. Sherry Rogers for technical assistance and $\mathrm{Mr}$. William Brudon for the artwork and gross photography.

\section{LITERATURE CITED}

Bischler, V. 1926 L'influence du squelette dans la régénération, et les potentialités des divers territoires du membre chez Triton cristatus. Rev. Suisse Zool., 33: 431-560.

Bryant, S. V., and L. E. Iten 1976 Supernumerary limbs in amphibians: experimental production in Notophthalmus viridescens and a new interpretation of their formation. Devel. Biol., 50: 212-234.

Burdi, A. R. 1965 Toluidine blue-alizarin red S staining of cartilage and bone in whole-mount skeletons in vitro. Stain Technol, 40: 45-48.

Chandebois, R. 1976 Histogenesis and Morphogenesis in Planarian Regeneration. Monographs in Developmental Biology. Vol. 11. S. Karger AG, Basel.

Globus, M., and R. A. Liversage 1975 In vitro studies of limb regeneration in adult Diemictylus viridescens: Neural dependence of blastemal cells for growth and differen. tiation. J. Embryol. Exp. Morph., 33: 813-829.

Goss, R. J. 1957 The relation of skin to defect regulation in regenerating half limbs. J. Morph., 100: 547-564.

Hsu, L. 1971 The role of nerves in the regeneration of minced muscle in adult anurans. Ph.D. Thesis, Univer. sity of Michigan, Ann Arbor.

Iten, L. E., and S. V. Bryant 1975 The interaction between the blastema and stump in the establishment of the anterior-posterior and proximal-distal organization of the limb regenerate. Devel. Biol., 44: 119-147.

Lheureux, E. 1975 Régénération des membres irradies de Pleurodeles waltlii Michah (Urodele). Influence des qualités et orientations des greffons non irradies. Wilhelm Roux' Arch., 176: 303-327.

Lomovskaya, E. G. 1948 The regeneration of axolotl limbs after irradiation of halves of them by Roentgen rays. Doklady Akad. Nauk SSSR, 61: 157.160 (in Russian).

Mescher, A. L. 1976 Effects on adult newt limb regeneration of partial and complete skin flaps over the amputation surface. J. Exp. Zool., 195: 117-128.

Morgan, T. H. 1901 Regeneration. The MacMillan Co., N.Y.

Rose, S. M. 1957 Polarized inhibitory effects during regeneration in Tubularia. J. Morph., 100: 187-205.

1962 Tissue-arc control of regeneration in the amphibian limb. In: Regeneration. D. Rudnick, ed. Ronald Press, N.Y., pp. 153-176.

Shuraleff, N. C., and C. S. Thornton 1967 An analysis of distal dominance in the regenerating limb of the axolotl. Experientia, 23: 747-748.

Stocum, D. L. 1975 Regulation after proximal or distal transposition of limb regeneration blastemas and determination of the proximal boundary of the regenerate. Devel. Biol., 45: 112-136.

Thornton, C. S, 1968 Amphibian limb regeneration. Adv. Morphogenesis, 7: 205-250.

Thornton, C. S., and D. W. Kraemer 1951 The effect of injury on denervated unamputated forelimbs of Amblys. toma larvae. J. Exp. Zool., 117: 415-440.

Tornier, G. 1896 Über Hyperdaktylie, Regeneration und Vererbung mit Experimenten. Arch. f. Entw.-mech., 3: 469.476.

Warkany, J. 1971 Congenital Malformations. Year Book Medical Publishers, Chicago.

Weiss, P. 1926 Ganzregenerate aus halbem Extremitätenquerschnitt. Arch. f. Entw.-mech., 107: 1-53. 
PLATE 1

\section{EXPLANATION OF FIGURES}

8 Normal 8-day regenerate. The amputation surface is covered by a somewhat thickened wound epidermis and the dedifferentiation phase is just beginning. The level of amputation is indicated by arrows. $\mathrm{H} \& \mathrm{E}$.

9 Contralateral limb of that shown in figure 8, eight days after amputation. This limb contains a digital implant (arrow, and all skeletal structures shown). The implant is well in tegrated in to the limb, with very little cellular reaction around it. The muscle fibers alongside the deep parts of the implant are somewhat disoriented. The ulnar side of the limb (right) is covered by a wound epidermis (e). H \& E.

10 Normal 25-day forearm regenerate, showing an undifferentiated cone regenerate. $\mathrm{H}$ \& $\mathrm{E}$.

11 Contralateral limb of the same newt shown in figure 10. This limb has a prominent digital implant that is well healed in place. On the ulnar side of the digit is an area of thickened wound epidermis (between arrows). Beneath it there are no indications of epimorphic regenerative activity, but rather prominent tissue healing reactions. $H \& E$. 

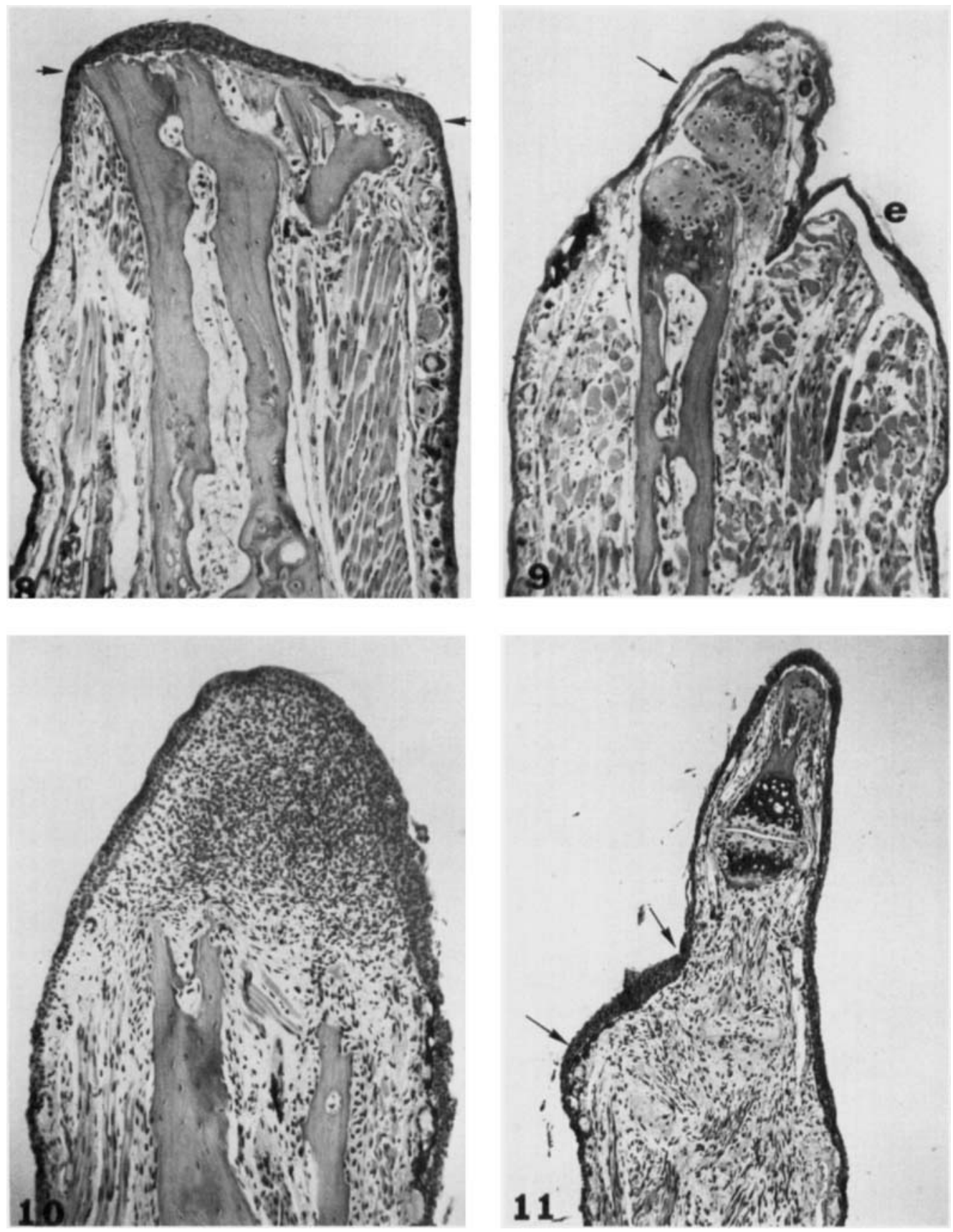
12 Normal 32-day forearm regenerate showing the early differentiation of skeletal elements. Prominent cuffs of well oriented periosteal cartilage surround the distal radius and ulna. $\mathrm{H} \& \mathrm{E}$.

13 Amputated forearm from the same newt as that shown in figure 12. This limb contains a digital implant (d). A blastema has not formed. Instead, a fibrocellular scar has formed beneath the ulnar wound epidermis. In addition, the cartilaginous callus forming distal to the ulna is radially deviated (arrow). H \& E.

14 Normal 39-day forearm regenerate, showing good differentiation of the skeletal elements and early differentiation of muscle. H \& $\mathbf{E}$.

15 Contralateral limb to that in figure 14. On the ulnar side of the digital implant (d) is a thickened wound epidermis (e) with a small aggregation of blastemal cells (b) beneath it. $\mathrm{H} \& \mathrm{E}$. 

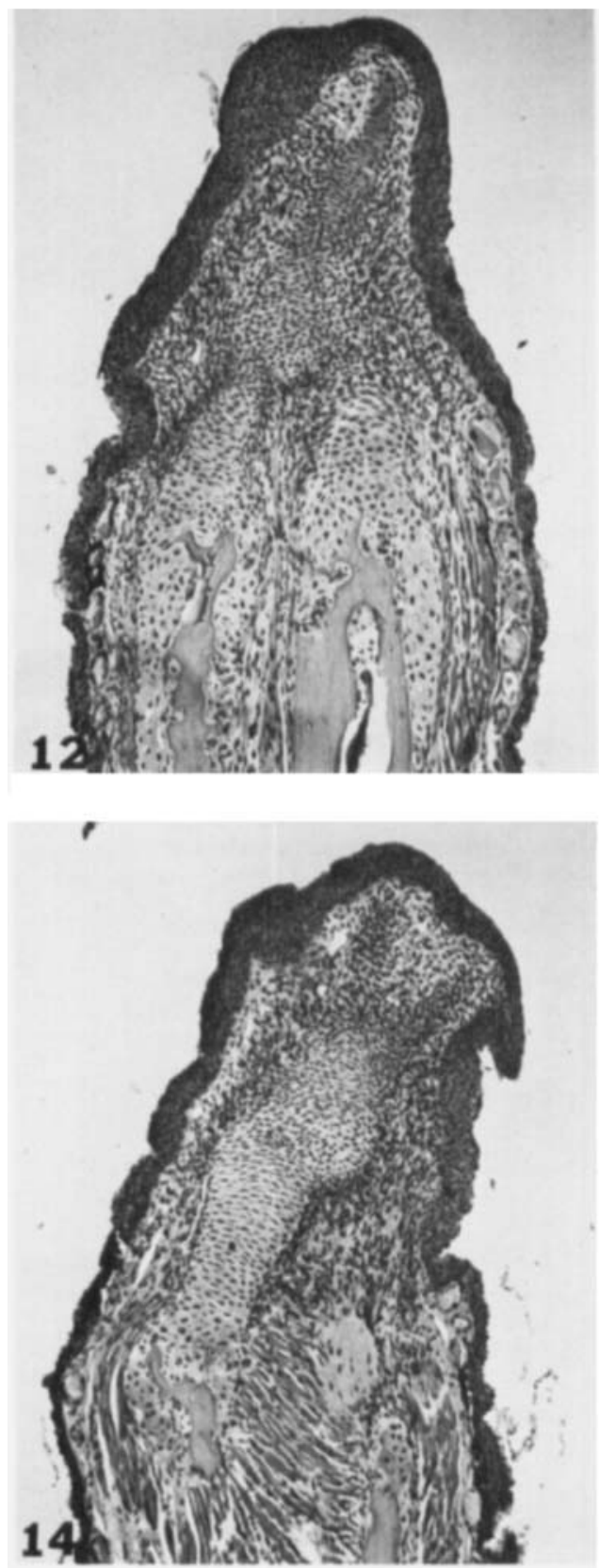
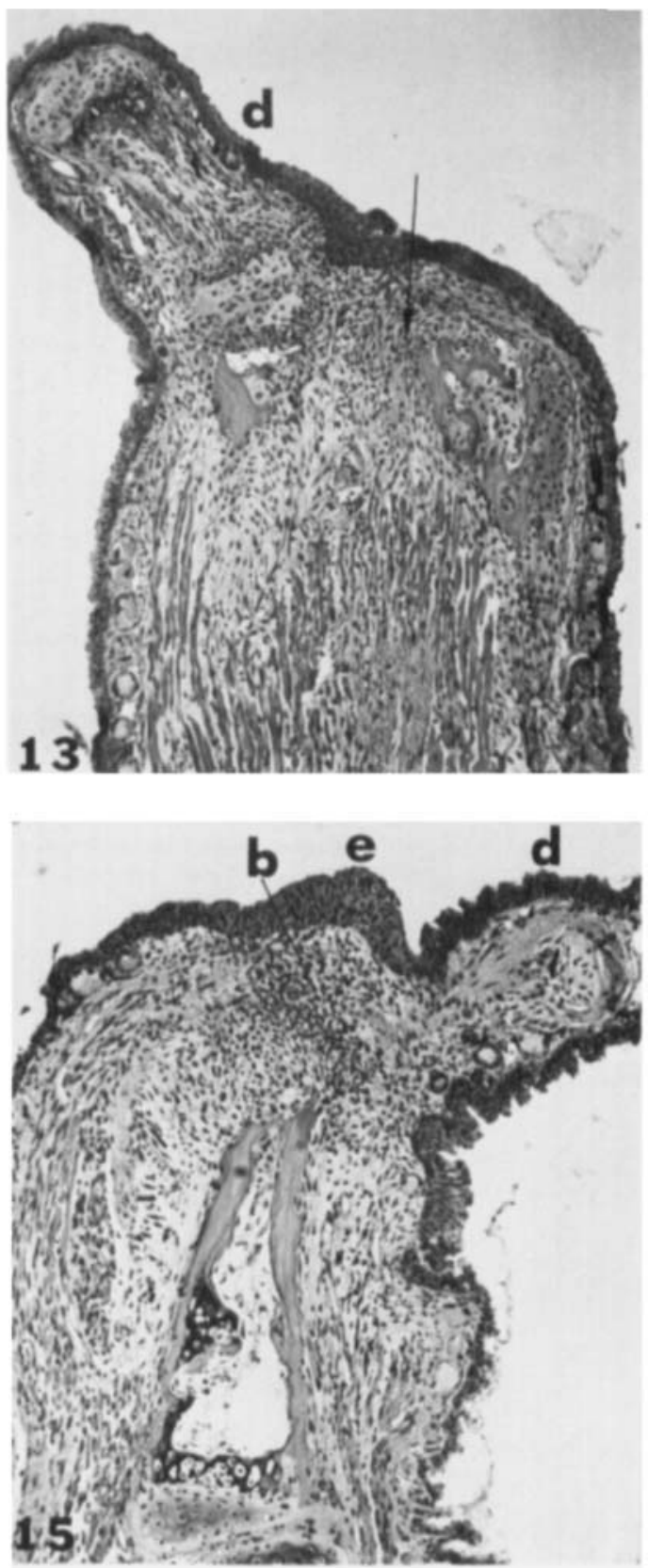
PLATE 3

EXPLANATION OF FIGURES

16 Normal 41-day forearm regenerate, showing all four digits and the differentiation of muscle fibers throughout the palmar area. $\mathrm{H} \& \mathrm{E}$.

17 Contralateral limb to that shown in figure 16. The digital implant (d) is well healed in place. Despite the presence of a large expanse of wound epidermis (between arrows), the limb shows no signs of epimorphic regenerative activity. $H \& \mathrm{E}$.

18 Non-regenerating forearm stump, bearing digital implant (d), 66 days after amputation. A fibrocellular scar (s) has formed between the distal end of the ulna and the overlying wound epidermis. A cartilaginous mass (c) has grown out perpendicularly to the ulna, toward the radial side of the limb. $H \& E$.

19 Nineteen-day regenerate in forearm from which the radius was removed. All regenerates in this series were deviated sharply toward the radial side. Neither the regenerating nerve trunk ( $n$ ) nor the apical epidermal thickening (e) showed significant radial deviation. Palmgren's silver stain. 

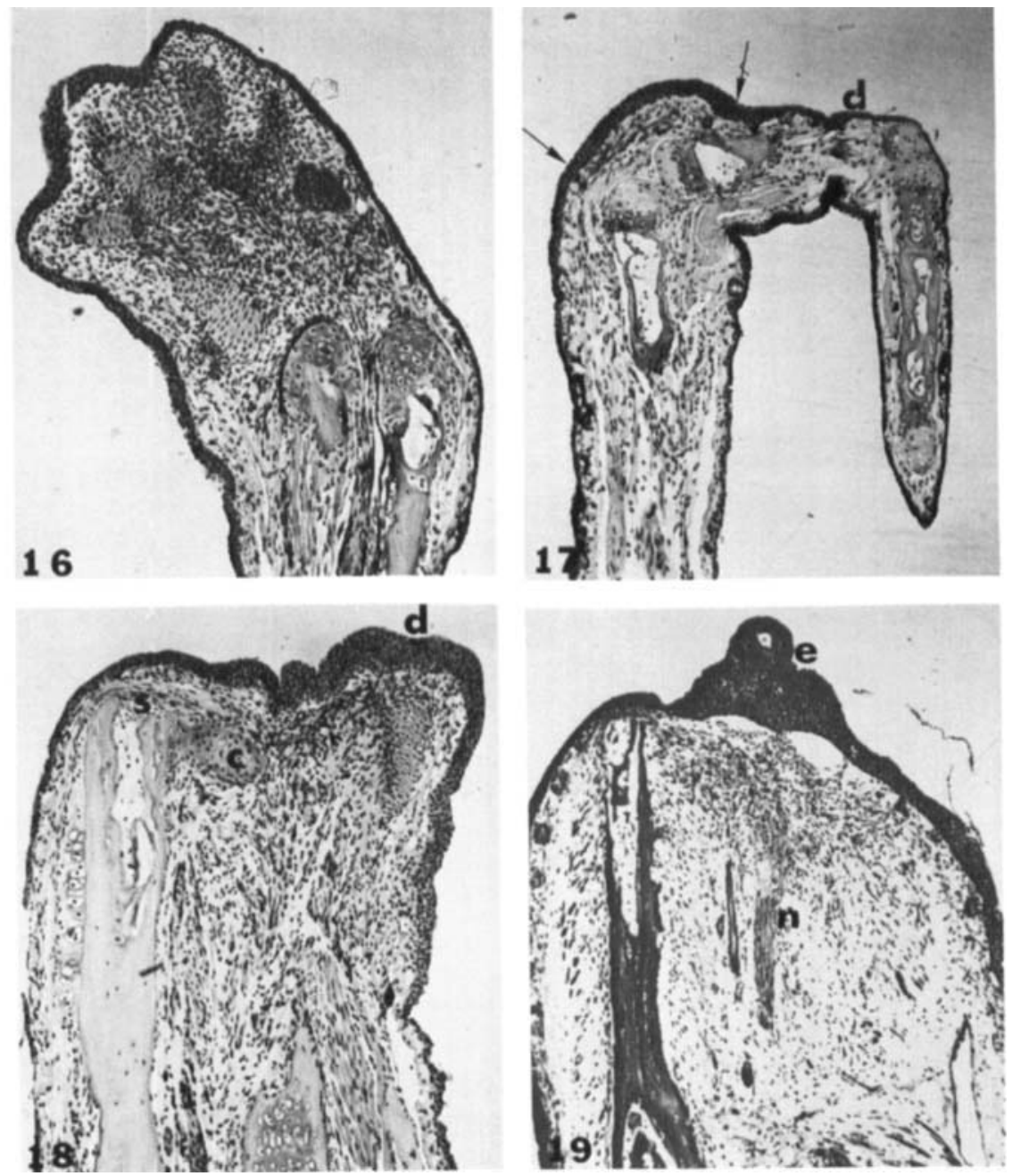